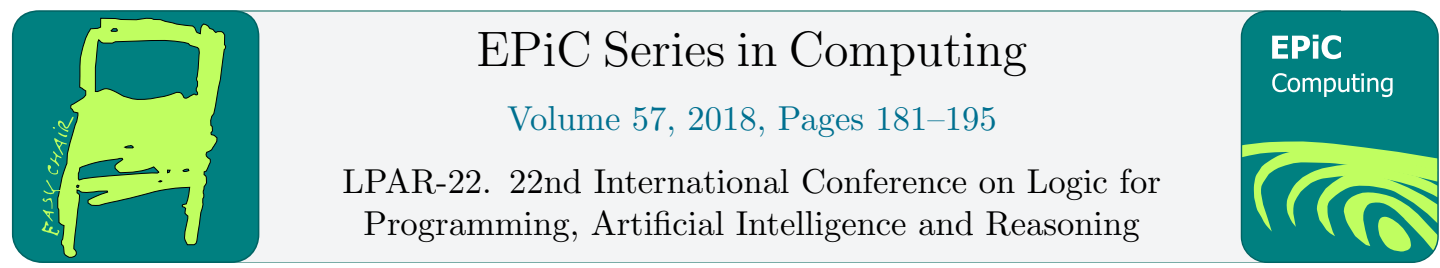

\title{
Efficient SAT-Based Encodings of Conditional Cardinality Constraints
}

\author{
Abdelhamid Boudane ${ }^{1}$, Said Jabbour ${ }^{1}$, Badran Raddaoui ${ }^{2}$, and Lakhdar Sais ${ }^{1}$ \\ 1 CRIL - CNRS, Artois University, Rue Jean Souvraz, SP-18, F-62307, Cedex, France \\ \{boudane, jabbour, sais\}@cril.fr \\ 2 SAMOVAR, Télécom SudParis, CNRS, Paris-Saclay University, F-91011, Evry Cedex, France \\ badran.raddaoui@telecom-sudparis.eu
}

\begin{abstract}
In the encoding of many real-world problems to propositional satisfiability, the cardinality constraint is a recurrent constraint that needs to be managed effectively. Several efficient encodings have been proposed while missing that such a constraint can be involved in a more general propositional formula. To avoid combinatorial explosion, the Tseitin principle usually used to translate such general propositional formula to Conjunctive Normal Form (CNF), introduces fresh propositional variables to represent sub-formulas and/or complex contraints. Thanks to Plaisted and Greenbaum improvement, the polarity of the sub-formula $\Phi$ is taken into account leading to conditional constraints of the form $y \rightarrow \Phi$, or $\Phi \rightarrow y$, where $y$ is a fresh propositional variable. In the case where $\Phi$ represents a cardinality constraint, such translation leads to conditional cardinality constraints subject of the present paper. We first show that when all the clauses encoding the cardinality constraint are augmented with an additional new variable, most of the well-known encodings cease to maintain the generalized arc-consistency property. Then, we consider some of these encodings and show how they can be extended to recover such important property. An experimental validation is conducted on a SAT-based pattern mining application, where such conditional cardinality constraints are a cornerstone, showing the relevance of our proposed approach.
\end{abstract}

\section{Introduction}

Continuous improvements in SAT solver technology have resulted in a real scaling up and widening of the class of real-world problems that can be solved in practice. The modeling phase of such increasing number and more complex applications into propositional formulas in Conjunctive Normal Form (CNF) suitable for use by a satisfiability solver becomes even more crucial. The modeling issue follows several polynomial transformations and rewriting steps, starting from high level description, using high order language or full propositional logic, to low level formulation, usually a formula in CNF. The whole process preserves satisfiability, thanks to the extension principle [16], allowing the introduction of new variables to represent sub-formulas or complex constraints. Among such constraints, cardinality and pseudo-boolean constraints, expressing numerical bounds on discrete quantities, are the most popular as they

G. Barthe, G. Sutcliffe and M. Veanes (eds.), LPAR-22 (EPiC Series in Computing, vol. 57), pp. 181-195 
arise frequently in the encoding of many real-world problems including scheduling, logic synthesis or verification, product configuration and data mining. For the above reasons, there have been various approaches addressing the issue of finding an efficient encoding of cardinality (e.g. $[17,3,15,13,1,11])$ and pseudo-boolean constraints (e.g. [8, 4]) as a CNF formula. Efficiency refers to both the compactness of the representation (size of the CNF formula) and to the ability to achieve the same level of constraint propagation (generalized arc consistency) on the CNF formula. However, most of the proposed encodings do not take care of the interactions between the cardinality constraint and the remaining part of the propositional formula, through different logical connectives. To avoid combinatorial explosion, the Tseitin principle [16] is usually used to translate general propositional formula to CNF, making use of fresh propositional variables to represent sub-formulas and/or complex contraints. Thanks to Plaisted and Greenbaum [14] improvement, the polarity of the sub-formula $\Phi$ is taken into account leading to conditional constraints of the form $y \rightarrow \Phi$ or $\Phi \rightarrow y$, where $y$ is a fresh propositional variable. When a cardinality constraint is involved as a sub-formula, such translation leads to what we call a conditional cardinality constraint. Such kind of constraints usually appear when encoding real world problems to CNF. For example in [9], the authors used conditional cardinality constraints as part of the clock routing problem encoding. The encoding to CNF was done by adding a selector on top of an existing encoding. They used many of such constraints in interaction with other bit-vector constraints. However, in [9], the authors do not show how to add selectors in a way that maintains generalized arc consistency. The clock routing application of [9] provides an additional evidence for the potential usefulness of the issue addressed in this paper.

In this paper, we investigate the SAT encoding of conditional cardinality constraints, defined as $y \rightarrow \sum_{i=1}^{n} x_{i} \leq k$. It expresses that no more than $k$ variables can be set to true, when setting the condition $y$ to true. We first show that by adding $\neg y$ disjunctively to all the clauses resulting from the encoding of the cardinality constraint, most of the well-known encodings cease to maintain constraint propagation. We then address the issue of extending such encodings while maintaining generalized arc consistency. We also consider the particular case of conditional AtMostOne constraints, i.e., $k=1$. An experimental evaluation is conducted on a SAT based non redundant association rules mining problem, showing the relevance of our proposed framework.

\section{Technical Background and Preliminary Definitions}

\subsection{Preliminary Definitions and Notations}

Let $\mathcal{L}$ be a propositional language of formulas built in the standard way, using usual logical connectives $(\vee, \wedge, \neg, \rightarrow, \leftrightarrow)$ and a set of propositional variables. A propositional formula $\mathcal{F}$ in CNF is a conjunction of clauses, where a clause is a disjunction of literals. A literal is a positive $(x)$ or a negated $(\neg x)$ propositional variable. A clause can be represented as a set of literals and a formula as a set of clauses. The two literals $x$ and $\neg x$ are called complementary. We note $\bar{l}$ the complementary literal of $l$. For a set of literals $L, \bar{L}$ is defined as $\{\bar{l} \mid l \in L\}$. For a clause $c$, we note $\bar{c}=\bigwedge_{l \in c} \bar{l}$. A unit clause is a clause containing only one literal (called unit literal), while a binary clause contains exactly two literals. A Horn (resp. reverse Horn) clause is a clause with at-most one positive (resp. negative) literal. A positive (resp. negative) clause is a clause whose literals are all positive (resp. negative). An empty clause, denoted $\perp$, is interpreted as false (unsatisfiable), whereas an empty CNF formula, denoted $T$, is interpreted as true (satisfiable).

Let us recall that any general propositional formula can be translated to CNF using linear 
Tseitin's encoding [16]. This can be done by introducing fresh variables to represent subformulas in order to represent their truth values. For example, given a propositional formula containing the variables $x$ and $y$, and $\alpha$ is a fresh variable, one can add the definition $\alpha \leftrightarrow$ $x \vee y$ (called extension) to the formula while preserving satisfiability. Two decades later, after Tseitin's seminal paper, Plaisted and Greenbaum presented an improved CNF translation that essentially produces a subset of Tseitin's representation [14]. The authors noticed that by keeping track of polarities of sub-formulas, one can remove large parts of Tseitin translation. For example, when the disjunction $x \vee y$ is a sub-formula with positive polarity, it is sufficient to add the formula $\alpha \rightarrow x \vee y$, i.e., a clause $(\neg \alpha \vee x \vee y)$.

The set of variables occurring in $\mathcal{F}$ is denoted $V_{\mathcal{F}}$ and its associated set of literals $L_{\mathcal{F}}=$ $\cup_{x \in \mathcal{V}_{\mathcal{F}}}\{x, \neg x\}$. A set of literals is complete if it contains one literal for each variable in $V_{\mathcal{F}}$, and fundamental if it does not contain complementary literals. A literal $l$ is called monotone or pure if $\tilde{l}$ does not appear in $\mathcal{F}$. An interpretation $\rho$ of a formula $\mathcal{F}$ is a function which associates a truth value $\rho(x) \in\{0,1\}$ ( 0 for false and 1 for true) to some of the variables $x \in V_{\mathcal{F}} . \rho$ is complete if it assigns a value to every variable $x \in V_{\mathcal{F}}$, and partial otherwise. An interpretation is alternatively represented by a complete and fundamental set of literals. A model of a formula $\mathcal{F}$ is an interpretation $\rho$ that satisfies the formula $(\rho(\mathcal{F})=1)$, denoted $\rho \models \mathcal{F}$. A formula $\mathcal{G}$ is a logical consequence of a formula $\mathcal{F}$, denoted $\mathcal{F} \models \mathcal{G}$, iff every model of $\mathcal{F}$ is a model of $\mathcal{G}$. The SAT problem consists in deciding if a given CNF formula admits a model or not.

$\left.\mathcal{F}\right|_{x}$ denotes the formula obtained from $\mathcal{F}$ by assigning $x$ the truth-value true. Formally, $\left.\mathcal{F}\right|_{x}=\{c \mid c \in \mathcal{F},\{x, \neg x\} \cap c=\emptyset\} \cup\{c \backslash\{\neg x\} \mid c \in \mathcal{F}, \neg x \in c\}$. This notation is extended to interpretations: given an interpretation $\rho=\left\{x_{1}, \ldots, x_{n}\right\}$, we define $\left.\mathcal{F}\right|_{\rho}=\left(\left.\ldots\left(\left.\left(\left.\mathcal{F}\right|_{x_{1}}\right)\right|_{x_{2}}\right) \ldots\right|_{x_{n}}\right)$. $\mathcal{F}^{*}$ denotes the formula $\mathcal{F}$ closed under unit propagation, defined recursively as follows: (1) $\mathcal{F}^{*}=\mathcal{F}$ if $\mathcal{F}$ does not contain any unit clause, $(2) \mathcal{F}^{*}=\perp$ if $\mathcal{F}$ contains two unit-clauses $\{x\}$ and $\{\neg x\},(3)$ otherwise, $\mathcal{F}^{*}=\left(\left.\mathcal{F}\right|_{x}\right)^{*}$ where $x$ is the literal appearing in a unit clause of $\mathcal{F}$. A clause $c$ is deduced by unit propagation from $\mathcal{F}$, noted $\mathcal{F}=^{*} c$, iff $(\mathcal{F} \wedge \bar{c})^{*}=\perp$. We define $\eta\left[x, c_{i}, c_{j}\right]$ as the resolvent between a clause $c_{i}$ containing the literal $x$ and $c_{j}$ a clause containing the literal $\neg x$. In other words $\eta\left[x, c_{i}, c_{j}\right]=\left(c_{i} \cup c_{j}\right) \backslash\{x, \neg x\}$. Resolution is the process of deriving a resolvent from two parents clauses containing two complementary literals.

\subsection{CNF Encodings of Cardinality Constraints: An Overview}

In this section, we overview most of the well-known encodings of cardinality constraints of the form $\sum_{i=1}^{n} x_{i} \leqslant k$, called AtMostK constraints. For clarity and consistency reasons, and as the constraint AtMostK $\left(\sum_{i=1}^{n} x_{i} \leqslant k\right)$ can be equivalently rewritten as an AtLeastK constraint $\sum_{i=1}^{n} \neg x_{i} \geqslant n-k$, for the pigeon hole based encoding, we consider the conditional AtLeastK constraint $y \rightarrow \sum_{i=1}^{m} x_{i} \geqslant k$.

\subsubsection{Pigeon-Hole based Encoding}

In [11], the authors proposed a new encoding of the cardinality constraints $\sum_{i=1}^{n} x_{i} \geq k$, based on the Pigeon-Hole principle. They observed that the semantic of the cardinality constraint can be equivalently expressed as the problem of putting $k$ pigeons into $n$ holes. The first formulation, called $\mathcal{P}_{n}^{k}$, given in [11], is simply expressed by the following set of constraints:

$$
\bigwedge_{j=1}^{k}\left(\neg p_{j i} \vee x_{i}\right), \quad 1 \leqslant i \leqslant n
$$




$$
\begin{gathered}
\bigvee_{i=1}^{n} p_{j i}, \quad 1 \leqslant j \leqslant k \\
\bigwedge_{1 \leqslant j<j^{\prime} \leqslant k}\left(\neg p_{j i} \vee \neg p_{j^{\prime} i}\right), \quad 1 \leqslant i \leqslant n
\end{gathered}
$$

The equations (2) and (3) encode the well-known pigeon hole problem $P H P_{n}^{k}$, where $k$ is the number of pigeons and $n$ is the number of holes $\left(p_{j i}\right.$ expresses that pigeon $j$ is in hole $i$ ). Unfortunately, checking the satisfiability of a Pigeon-Hole formula is computationally hard for SAT solvers, except if we use resolution with symmetry or extended resolution proof systems. To maintain generalized arc consistency (GAC), the authors proposed an improvement obtained by breaking the symmetries between the variables $p_{i j}$ involved in the pigeon hole expression. By resolution between the clauses of symmetry breaking predicates and those of $\mathcal{P}_{n}^{k}$, the authors derived the following encoding, called $p h \mathcal{P}_{n}^{k}$ :

$$
\begin{gathered}
\bigwedge_{1 \leq i \leq k}\left(\bigvee_{1 \leq j \leq n-k+1} p_{i j}\right) \\
\left.\bigwedge_{1 \leq i \leq k, 1 \leq j \leq n-k+1}\left(x_{i+j-1} \vee \neg p_{i j}\right)\right) \\
\left.\bigwedge_{1 \leq i<k, 1 \leq j<n-k+1}\left(\neg p_{(i+1) j} \vee \bigvee_{1 \leq l \leq j} p_{i l}\right)\right)
\end{gathered}
$$

Example 1. Let us consider the inequality $x_{1}+x_{2}+x_{3}+x_{4}+x_{5}+x_{6} \geq 4$. Using the pigeon-hole based encoding $p h \mathcal{P}_{6}^{4}$, we obtain the following $C N F$ :

$\begin{array}{llll}p_{11} \vee \neg p_{21} & x_{1} \vee \neg p_{11} & x_{2} \vee \neg p_{12} & \\ p_{11} \vee p_{12} \vee \neg p_{22} & x_{2} \vee \neg p_{21} & x_{3} \vee \neg p_{13} & p_{11} \vee p_{12} \vee p_{13} \\ p_{21} \vee \neg p_{31} & x_{3} \vee \neg p_{22} & x_{3} \vee \neg p_{31} & p_{21} \vee p_{22} \vee p_{23} \\ p_{21} \vee p_{22} \vee \neg p_{32} & x_{4} \vee \neg p_{23} & x_{4} \vee \neg p_{32} & p_{31} \vee p_{32} \vee p_{33} \\ p_{31} \vee \neg p_{41} & x_{4} \vee \neg p_{41} & x_{5} \vee \neg p_{33} & p_{41} \vee p_{42} \vee p_{43} \\ p_{31} \vee p_{32} \vee \neg p_{42} & x_{5} \vee \neg p_{42} & x_{6} \vee \neg p_{43} & \end{array}$

\subsubsection{Sorting Networks based Encoding}

One of the most effective encodings for cardinality constraints is based on sorting networks [8]. In this encoding, the cardinality constraint $\sum_{i=1}^{n} x_{i} \leqslant k$ is translated into a single sorter with $n$ inputs $X=\left\{x_{1}, \ldots, x_{n}\right\}$ and $n$ outputs $Z=\left\{z_{1}, \ldots, z_{n}\right\}$ (sorted in descending order) where the $\mathrm{k}^{\text {th }}$ output is forced to true. The idea behind this encoding is to sort the input variables into true variables followed by false variables. To satisfy the constraint $\sum_{i=1}^{n} x_{i} \leqslant k$, it is sufficient to set $z_{k+1}$ to false. In [8], the authors proved that the sorting networks based encoding maintains generalized arc consistency. Let us note $\Phi_{S N}^{n, k}(X ; Z)$ the formula representing the sorting networks based circuit that takes as input the set of propositional variables $X$ and outputs an unary number represented by the set of propositional variables $Z$. The following formula defines the encoding:

$$
\Phi_{S N}^{n, k}(X ; Z) \wedge \neg z_{k+1}
$$

As the outputs $Z$ are sorted in descending order, by fixing $z_{k+1}$ to false, all the remaining variables $z_{k+2}, \ldots, z_{n}$ must be propagated to false. Consequently, as the output variables are 
sorted in descending order, at most $k$ variables $z_{1}, \ldots, z_{k}$ might be assigned to true. Let us note that the formula $\Phi_{S N}^{n, k}(X ; Z)$ encoding the sorting network is a Horn formula, derived using a basic comparator between two propositional variables [8]. Given two propositional variables $x_{1}$ and $x_{2}$ from $X$, the comparator outputs two variables $z_{1}$ and $z_{2}$ from $Z$, the two comparator, noted 2-comp $\left(x_{1}, x_{2} ; z_{1}, z_{2}\right)$, is defined by the following Horn formula:

$$
x_{1} \rightarrow z_{1} ; x_{2} \rightarrow z_{1} ; x_{1} \wedge x_{2} \rightarrow z_{2}
$$

This formula allows to sort the two variables $x_{1}$ and $x_{2}$ resulting in two other variables $z_{1}$ and $z_{2}$ in descending order. For example, when $x_{1}$ (resp. $x_{2}$ ) is assigned to false (resp. true), the output variable $z_{1}$ (resp. $z_{2}$ ) is assigned to true (resp. false). For more details, we refer the reader to [8] and [2].

\subsubsection{Sequential Unary Counter based Encoding}

The sequential counter based encoding of a cardinality constraint proposed by Carsten Sinz in [15] is another well-known encoding that preserves the generalized arc consistency property. It computes for each propositional variable $x_{i}$, the partial sums $s_{i}=\sum_{j=1}^{i} x_{j}$ for $1 \leqslant i \leqslant n$. The values of all the sums are represented as unary numbers of size equals to $k$. The encoding is defined as follows:

$$
\begin{gathered}
\left(\neg x_{1} \vee s_{1,1}\right) \\
\bigwedge_{1<j \leqslant k} \neg s_{1, j} \\
\bigwedge_{1<i<n}\left(\neg x_{i} \vee s_{i, 1}\right) \wedge\left(\neg s_{i-1,1} \vee s_{i, 1}\right) \\
\bigwedge_{1<j \leqslant k}\left(\neg x_{i} \vee \neg s_{i-1, j-1} \vee s_{i, j}\right) \wedge\left(\neg s_{i-1, j} \vee s_{i, j}\right) \\
\bigwedge_{1<i \leqslant n}\left(\neg x_{i} \vee \neg s_{i-1, k}\right)
\end{gathered}
$$

The variables $s_{i, j}$ denotes the $j^{\text {th }}$ digit of the $i^{t h}$ partial sum $s_{i}$ in unary representation. The constraints (9) and (10) correspond to the case $i=1$. The formula (13) is very important. It allows to detect the inconsistency and preserves the GAC property at the same time. The other constraints allow the propagation of any changes of a partial sum $s_{i}$ after any assignment of $x_{i}$ variables. Let us note that the formula derived by the sequential unary counter based encoding is also a Horn formula.

\section{Conditional Cardinality Constraints Encodings}

In this section, we show how the cardinality constraint SAT-based encodings of Section 2.2, can be effectively extended to encode conditional cardinality constraints of the form $y \rightarrow \sum_{i=1}^{n} x_{i} \leqslant$ $k$ while preserving generalized arc consistency maintained by unit propagation. More precisely, for such conditional cardinality constraint, maintaining GAC, means that when $y$ is assigned the truth value true, the encoding must maintain GAC on the cardinality constraint $\sum_{i=1}^{n} x_{i} \leqslant k$. On the other hand, when the cardinality constraint is false under the current assignment, the literal $\neg y$ must be deduced by unit propagation. 
An important observation that can be made from the SAT based encodings of the cardinality constraint presented in the previous subsection, is that the obtained formula is Horn (or reverse Horn). Let us first introduce an important property, allowing us to grasp the intuition behind the encodings we propose in this paper.

Let $\Phi$ be a Horn formula, the sub-formula $\Phi^{-}$denotes the set of negative clauses of $\Phi$ and $\Phi^{+}$the set of clauses of $\Phi$ containing exactly one positive literal.

Proposition 1. Let $\Phi$ be a Horn formula and $\rho=\left\{x_{1}, x_{2}, \ldots, x_{k}\right\} \subseteq V_{\Phi}$ an interpretation. Then $\left.\Phi\right|_{\rho} \models^{*} \perp$ iff $\exists c \in \Phi^{-}$such that $\left.\left.\Phi^{+}\right|_{\rho}\right|^{*} \bar{c}$.

Proof. $(\Rightarrow)$ Let us consider the formula $\left.\Phi^{+}\right|_{\rho}$. Suppose that there is no clause $c \in \Phi^{-}$such that $\left.\Phi^{+}\right|_{\rho} \models^{*} \bar{c}$. Let $S$ be the set of units literals of $\left.\Phi^{+}\right|_{\rho}$ including the literals of $\rho$. We can note that from $\left.\Phi^{+}\right|_{\rho}$ only additional positive unit literals can be deduced by unit propagation $(S \supseteq \rho)$.

So $S$ is a set of positive literals. $S \cup \overline{(V(\Phi) \backslash S)}$ is clearly a model of $\left.\Phi\right|_{\rho}$. In fact, each clause of $\left.\Phi^{+}\right|_{\rho}$ is a satisfied clause (its positive literal is in $S$ ) or contains at least one negative literal. Indeed, propagating positive literals over $\Phi^{+}$leads to a formula where the remaining clauses contain a positive literal and at least one negative literal. The remaining clauses of $\Phi^{-} \wedge S$ are negative clauses before deleting from each clause the literals of $\bar{S}$. Then, by assigning the remaining variables $V(\Phi) \backslash S$ to false, we obtain a model $\mu=S \cup \overline{(V(\Phi) \backslash S)}$ of the formula $\Phi$. As $\rho \subseteq \mu$, this contradicts the assumption that $\left.\Phi\right|_{\rho}$ is unsatisfiable.

$(\Leftarrow)$ From $\left.\Phi^{+}\right|_{\rho} \models^{*} \bar{c}$, we have $\left.\left.\left(\Phi^{+} \wedge c\right)\right|_{\rho}\right|^{*} \perp$. As $\Phi^{+} \wedge c \subseteq \Phi$, then $\left.\left.\Phi\right|_{\rho}\right|^{*} \perp$.

Given a Horn formula $\Phi$, Proposition 1 expresses that unsatisfiability under any interpretation made of a set of positives literals, is caused by a clause from $\Phi^{-}$. As a cardinality constraint is usually encoded as a Horn formula $\Phi$, to maintain GAC on the encoding of $y \rightarrow \Phi$, one only need to disjunctively add $\neg y$ to $\Phi^{-}$.

\subsection{Conditional AtMostOne Constraint Encodings}

Let us first consider the conditional AtMostOne Constraint $y \rightarrow \sum_{i=1}^{n} x_{i} \leq 1$. Many encodings have been proposed to deal with the translation of AtMostOne constraint into CNF. Let us consider two standard encodings of this constraint.

\subsubsection{Conditional AtMostOne Pairwise Encoding}

The classical pairwise encoding can be obtained by considering the set of all binary negative clauses build over the set of variables $\left\{x_{1}, \ldots, x_{n}\right\}$ as described by the formula (14).

$$
\bigwedge_{1 \leq i<j \leq n}\left(\neg x_{i} \vee \neg x_{j}\right)
$$

This naive formulation maintains generalized arc consistency and is in $\mathcal{O}(n)$ variables and $\mathcal{O}\left(n^{2}\right)$ clauses. The formula (15) encoding the conditional AtMostOne constraint $y \rightarrow \sum_{i=1}^{n} x_{i} \leq$ 1 is obtained by simply adding $\neg y$ to all the clauses of the CNF formula (14) obtained by pairwise encoding. It is straightforward to remark that the obtained formula (15) allows to maintain generalized arc consistency. Indeed, any assignment of two literals $x_{i}$ and $x_{j}$ to true, allows to deduce $\neg y$ by unit propagation. On the other hand, if $y$ is assigned to true, the conditional constraint is reduced to a simple AtMostOne constraint which preserve generalized arc consistency.

$$
\bigwedge_{1 \leq i<j \leq n}\left(\neg \mathbf{y} \vee \neg x_{i} \vee \neg x_{j}\right)
$$




\subsubsection{Conditional AtMostOne: Sequential Counter \& Pigeon-Hole Encodings}

The second encoding of the AtMostOne constraint is represented by formula (16) obtained using sequential counter [15]. In [11], the authors show that the same formula is obtained using the pigeon-hole encoding described above and by applying an additional step of variables elimination by resolution. In contrast to pairwise encoding (14), the one obtained by sequential counter (16) is linear $\left(\mathcal{O}(n)\right.$ variables and clauses) thanks to the additional variables $\left\{p_{1}, \ldots, p_{n-1}\right\}$. Both encodings (14) and (16) are known to maintain generalized arc consistency.

$$
\begin{aligned}
& \left(\neg x_{1} \vee p_{1}\right) \wedge\left(\neg x_{n} \vee \neg p_{n-1}\right) \wedge \\
& \bigwedge_{1<i<n}\left(\neg x_{i} \vee p_{i}\right) \wedge\left(\neg p_{i-1} \vee p_{i}\right) \wedge\left(\neg x_{i} \vee \neg p_{i-1}\right)
\end{aligned}
$$

However, with the sequential counter or Pigeon-Hole based encoding, by adding $\neg y$ to all clauses of the formula (16) we obtain a new formulation of the conditional AtMostOne constraint (formula (17)) that does not maintain generalized arc consistency.

$$
\begin{aligned}
& \left(\neg \mathbf{y} \vee \neg x_{1} \vee p_{1}\right) \wedge\left(\neg \mathbf{y} \vee \neg x_{n} \vee \neg p_{n-1}\right) \wedge \\
& \bigwedge_{1<i<n}\left(\neg \mathbf{y} \vee \neg x_{i} \vee p_{i}\right) \wedge\left(\neg \mathbf{y} \vee \neg p_{i-1} \vee p_{i}\right) \wedge\left(\neg \mathbf{y} \vee \neg x_{i} \vee \neg p_{i-1}\right)
\end{aligned}
$$

Indeed, assigning two literals from $\left\{x_{1}, \ldots, x_{n}\right\}$ does not allow us to deduce $\neg y$ by unit propagation. For example, by assigning $x_{1}$ and $x_{n}$ to true, the two first clauses from (17) become binary.

To maintain GAC for the conditional AtMostOne constraint using sequential counter or pigeon-hole based encoding, $\neg y$ must be added to a subset of the clauses as depicted in the formula (18).

$$
\begin{aligned}
& \left(\neg x_{1} \vee p_{1}\right) \wedge\left(\neg \mathbf{y} \vee \neg x_{n} \vee \neg p_{n-1}\right) \wedge \\
& \bigwedge_{1<i<n}\left(\neg x_{i} \vee p_{i}\right) \wedge\left(\neg p_{i-1} \vee p_{i}\right) \wedge\left(\neg \mathbf{y} \vee \neg x_{i} \vee \neg p_{i-1}\right)
\end{aligned}
$$

Proposition 2. The CNF formula (18) encoding $y \rightarrow \sum_{i=1}^{n} x_{i} \leq 1$ maintains generalized arc consistency by unit propagation.

Proof. The proof of this proposition is a direct consequence of Proposition 1. In fact, the encoding of $\sum_{i=1}^{n} x_{i} \leq 1$ is a Horn formula. As a consequence when more than one literal from $\left\{x_{1}, \ldots, x_{n}\right\}$ are assigned to true, then a clause from the negative clauses of the encodings become false. Consequently, to encode $y \rightarrow \sum_{i=1}^{n} x_{i} \leq 1$, it is sufficient to add $\neg y$ to the two negative clauses as shown in Constraint (18). Indeed, suppose that we assign two arbitrary variables $x_{i}$ and $x_{j}$ (with $1<i<j<n$ ) to true. From the assignment of $x_{i}$ to true and the clause $\left(\neg x_{i} \vee p_{i}\right)$, we deduce a unit literal $p_{i}$. Then, from the clause $\left(\neg p_{i} \vee p_{i+1}\right)$ we deduce another unit literal $p_{i+1}$. This chain of unit propagated literals continue until $p_{j-1}$. Now if we assign $x_{j}$ to true, the clause $\left(\neg y \vee \neg x_{j} \vee \neg p_{j-1}\right)$ allows us to deduce $\neg y$, as $p_{j-1}$ (propagated unit literal) and $x_{j}$ are assigned to true. Let us consider another case, where $x_{1}$ is assigned to true. Such assignment allows us to deduce thanks to unit propagation the literals $p_{1}, \ldots, p_{n-1}$. Then assigning any other literal $x_{j}$ (with $j \neq 1$ ), we deduce the literal $\neg y$, thanks to the clause $\left(\neg y \vee \neg x_{j} \vee \neg p_{j-1}\right)$. Obviously assigning $y$ to true leads to the classical encoding of the AtMostOne constraint which for the sequential counter or pigeon hole encoding preserves GAC by unit propagation. 


\subsubsection{Conditional AtMostOne: Sorting Networks Encoding}

The sorting network encoding of the AtMostOne conditional constraint is similar to the conditional AtMostK constraint described in Section 3.2.2. It is defined as:

$$
\Phi_{S N}^{n, 1}(X ; Z) \wedge\left(\neg \mathbf{y} \vee \neg z_{2}\right)
$$

Proposition 4 shows that the encoding, for any value of $k>0$, maintains generalized arc consistency by unit propagation.

\subsection{Conditional AtMostK Constraint Encodings}

Let us now consider the general case of Conditional AtMostK Constraint.

\subsubsection{Pigeon-Hole based Encoding of Conditional Cardinality}

In Subsection 2.2.1, we reviewed the pigeon hole based encoding of the cardinality constraint AtLeastK of the form $\sum_{i=1}^{n} x_{i} \geqslant k$ proposed in [11]. As the AtMostK constraint can be equivalently rewritten as AtLeastK constraint and vice-versa (see Section 2.2), for the pigeon hole based encoding, we consider the conditional AtLeastK constraint $y \rightarrow \sum_{i=1}^{n} x_{i} \geqslant k$.

To preserve GAC, $\neg y$ must be added to a limited subset of clauses of $p h \mathcal{P}_{n}^{k}$ encoding. Only the positives clauses of constraint (4) are augmented with $\neg y$.

$$
\bigwedge_{1 \leq i \leq k}\left(\neg y \vee \bigvee_{1 \leq j<n-k+1} p_{i j}\right)
$$

Proposition 3. The encoding $(19) \wedge(5) \wedge(6)$ preserves the generalized arc consistency of $y \rightarrow \sum_{i=1}^{n} x_{i} \geqslant k$.

Proof. (Sketch) Let us note that the pigeon hole based encoding of $\sum_{i=1}^{n} x_{i} \geqslant k$ is a reverseHorn formula. So the Proposition 1 can be slightly modified to be adapted to the reverse-Horn case by considering assignments of variables to false and positive clauses. As a consequence, one can conclude that adding $\neg y$ to the positive clauses is sufficient to maintain GAC by unit propagation. Let us sketch the proof using Example 1. The CNF encoding of the conditional constraint $y \rightarrow \sum_{i=1}^{6} x_{i} \geqslant 4$ is obtained from the CNF formula encoding $p h p \mathcal{P}_{6}^{4}$ by disjunctively adding $\neg y$ to the positive clauses (clauses on the right hand side). As we can observe the obtained formula remains in the reverse Horn class. Let us show that by assigning any three variables among $x_{1}$ to $x_{6}$ to false, we deduce $\neg y$ by unit propagation. Suppose that $x_{1}, x_{2}$ and $x_{3}$ are assigned to false. From the second and third set of clauses, we deduce by unit propagation $\neg p_{11}, \neg p_{12}, \neg p_{21}, \neg p_{13}, \neg p_{22}$ and $\neg p_{31}$. Consequently, from the clause $\left(\neg y \vee p_{11} \vee\right.$ $p_{12} \vee p_{13}$ ), we deduce $\neg y$. Let us consider another case, say $x_{2}, x_{4}$ and $x_{6}$ are assigned false. By unit propagation, we deduce $\neg p_{12}, \neg p_{21}, \neg p_{23}, \neg p_{32}, \neg p_{41}, \neg p_{43}, \neg p_{31}, \neg p_{41}, \neg p_{42}$. From the clause $\left(\neg y \vee p_{41} \vee p_{42} \vee p_{43}\right)$, we deduce $\neg y$. Similarly, any other assignment of three variables from $x_{1}$ to $x_{6}$ produces $\neg y$ by unit propagation.

\subsubsection{Sorting Networks based Encoding of Conditional Cardinality}

Let us now consider the sorting networks based encoding of the conditional AtMostK constraint $y \rightarrow \sum_{i=1}^{m} x_{i} \leqslant k$. Using the sorting networks encoding of the AtMostK constraint (see Section $2.2 .2)$, its conditional variant can be represented by $y \rightarrow\left(\Phi_{S N}^{n, k}(X ; Z) \wedge \neg z_{k+1}\right)$ which is equivalent to the CNF formula $\left(\neg \mathbf{y} \vee \Phi_{S N}^{n, k}(X ; Z)\right) \wedge\left(\neg \mathbf{y} \vee \neg z_{k+1}\right)$. As discussed in Section 2.2.2, the 
basic comparator of two propositional variables, $2-\operatorname{cmp}\left(x_{i}, x_{j} ; z_{i}, z_{j}\right)$, is a building bloc of the sorting networks based encoding $\Phi_{S N}^{n, k}(X ; Z)$, i.e., a conjunction of multiple formulas encoding two comparator basic components. Consequently, the conditional formula $\left(\neg y \vee \Phi_{S N}^{n, k}(X ; Z)\right)$ can be translated into CNF by adding $\neg y$ to all the clauses of each basic two comparators, which leads to multiple conditional two comparators of the form $y \rightarrow 2$-cmp $\left(x_{i}, x_{j} ; z_{i}, z_{j}\right)$, written in a clausal form as:

$$
\left(\neg \mathbf{y} \vee \neg x_{i} \vee z_{i}\right) \wedge\left(\neg \mathbf{y} \vee \neg x_{j} \vee z_{i}\right) \wedge\left(\neg \mathbf{y} \vee \neg x_{i} \vee \neg x_{j} \vee z_{j}\right)
$$

As we can see, assigning any input literal $x_{i}$ or $x_{j}$ to a conditional two comparators does not allow us to deduce any literal by unit propagation as all the clauses from (20) become binary. In fact, to maintain generalized arc consistency for the conditional AtMostK constraint using sorting networks-based encoding, $\neg y$ must be disjunctively added only to the unit clause $\neg z_{k+1}$ :

$$
\Phi_{S N}^{n, k}(X ; Z) \wedge\left(\neg \mathbf{y} \vee \neg z_{k+1}\right)
$$

Proposition 4. The encoding $\Phi_{S N}^{n, k}(X ; Z) \wedge\left(\neg \mathbf{y} \vee \neg z_{k+1}\right)$ preserves the generalized arc consistency of $y \rightarrow \sum_{i=1}^{n} x_{i} \leqslant k$.

Proof. In case where $y$ is assigned to true, the simplified formula represents the AtMostK constraint encoded using sorting networks. Now, we consider two cases depending on the truthvalue of $z_{k+1}$. In the first case, if $z_{k+1}$ is assigned to true, we deduce $\neg y$ by unit propagation. Indeed, as the outputs are sorted in descending order, this means that the AtMostK constraint is false, to satisfy the conditional AtMostK, one must assign $y$ to false. In the second case, if the truth value of $z_{k+1}$ is false, this means that the AtMostK constraint is true, consequently, no matter is the value of $y$.

\subsubsection{Sequential Unary Counter based Encoding of Conditional Cardinality}

We have shown in Subsection 3.1 how the conditional AtMostOne constraint can be encoded using the sequential counter-based encoding, while preserving the GAC property. Let us now consider the general case of Sequential counter-based encoding of conditional AtMostK constraint. The clauses $(9) \wedge(11) \wedge(12)$ allow us to propagate any assignment of $x_{i}$ to synchronize all the intermediate sequential counters, while the clauses $(10) \wedge(13)$ allow us to detect any inconsistency of the constraint AtMostK. Indeed, by adding $\neg y$ to all the clauses, the literals $s_{i j}$ can not be propagated from any assignment of $x_{i}$ variables which prevent the synchronization operation. To preserve the GAC property, we should add $\neg y$ only to the clauses of $(10) \wedge(13)$ as shown in the following formula:

$$
\begin{gathered}
\left(\neg x_{1} \vee s_{1,1}\right) \\
\bigwedge_{1<j \leqslant k}\left(\neg \mathbf{y} \vee \neg s_{1, j}\right) \\
\bigwedge_{1<i<n} \bigwedge_{1<j \leqslant k}\left(\neg x_{i} \vee s_{i, 1}\right) \wedge\left(\neg s_{i-1,1} \vee s_{i, 1}\right) \\
\bigwedge_{1<i \leqslant n}\left(\neg s_{i-1, j-1} \vee s_{i, j}\right) \wedge\left(\neg s_{i-1, j} \vee s_{i, j}\right)
\end{gathered}
$$


Example 2. Let us consider the following constraint $y \rightarrow x_{1}+x_{2}+x_{3} \leqslant 2$ which is encoded as follows:

$\left(\neg x_{1} \vee s_{1,1}\right) \wedge\left(\neg \mathbf{y} \vee \neg s_{1,2}\right) \wedge\left(\neg x_{2} \vee s_{2,1}\right) \wedge\left(\neg s_{1,1} \vee s_{2,1}\right) \wedge\left(\neg x_{2} \vee \neg s_{1,1} \vee s_{2,2}\right) \wedge\left(\neg s_{1,2} \vee s_{2,2}\right) \wedge$ $\left(\neg \mathbf{y} \vee \neg x_{2} \vee \neg s_{1,2}\right) \wedge\left(\neg \mathbf{y} \vee \neg x_{3} \vee \neg s_{2,2}\right)$

Assume that we start by assigning $x_{1}$ to true then, the literals $s_{1,1}$ and $s_{2,1}$ are deduced by unit propagation. Next, if we assign $x_{2}$ to true, the literal $s_{2,2}$ is unit propagated. Finally by assigning $x_{3}$ to true, which violates the constraint, the literal $\neg y$ is propagated thanks to the last clause.

Proposition 5. The encoding $(22) \wedge(23) \wedge(24) \wedge(25) \wedge(26)$ preserves the generalized arc consistency of $y \rightarrow \sum_{i=1}^{n} x_{i} \leqslant k$.

Proof. The encoding based on sequential counter of the cardinality constraint is also a Horn formula. Consequently, we can apply the result of Proposition 1 to conclude that $\neg y$ must be added only to the negative clauses in order to preserve the generalized arc consistency. The proof is a simple generalization of those sketched in Example 2.

\section{SAT-based Association Rules Mining: A Case Study}

We now present an application case, the problem of mining non-redundant association rules, whose encoding involves many conditional atMostOne constraints.

\subsection{Association Rules Mining}

Let $\Omega$ be a finite non empty set of symbols, called items. We use the letters $a, b$, $c$, etc. to range over the elements of $\Omega$. An itemset $I$ over $\Omega$ is defined as a subset of $\Omega$, i.e., $I \subseteq \Omega$. We use $2^{\Omega}$ to denote the set of itemsets over $\Omega$ and we use the capital letters $I, J, K$, etc. to range over the elements of $2^{\Omega}$. A transaction is an ordered pair $(i, I)$ where $i$ is a natural number, called transaction identifier, and $I$ an itemset, i.e., $(i, I) \in \mathbb{N} \times 2^{\Omega}$. A transaction database $\mathcal{D}$ is defined as a finite non empty set of transactions $\left(\mathcal{D} \subseteq \mathbb{N} \times 2^{\Omega}\right)$ where each transaction identifier refers to a unique itemset. The cover of an itemset $I$ in a transaction database $\mathcal{D}$ is defined as $\operatorname{Cover}(I, \mathcal{D})=\{i \in \mathbb{N} \mid(i, J) \in \mathcal{D}$ and $I \subseteq J\}$. The support of $I$ in $\mathcal{D}$ corresponds to the cardinality of $\operatorname{Cover}(I, \mathcal{D})$, i.e., $\operatorname{Support}(I, \mathcal{D})=|\operatorname{Cover}(I, \mathcal{D})|$. An itemset $I \subseteq \Omega$ such that $\operatorname{Support}(I, \mathcal{D}) \geqslant 1$ is a closed itemset if, for all itemsets $J$ with $I \subset J$, $\operatorname{Support}(J, \mathcal{D})<\operatorname{Support}(I, \mathcal{D})$.

Example 3. Let us consider the transaction database $\mathcal{D}$ depicted in Table 1. We have $\operatorname{Cover}(\{c, d\}, \mathcal{D})=\{1,2,3,4,5\}$ and Support $(\{c, d\}, \mathcal{D})=5$ while Support $(\{f\}, \mathcal{D})=3$. The itemset $\{c, d\}$ is closed, while $\{f\}$ is not.

\begin{tabular}{|c|c|}
\hline tid & Itemsets \\
\hline 1 & $\begin{array}{lllll}C & D & E & F & G\end{array}$ \\
\hline 2 & $\begin{array}{lllll}C & D & E & F & G\end{array}$ \\
\hline 3 & $\begin{array}{llll}A & B & C & D\end{array}$ \\
\hline 4 & $A \quad B \quad C \quad D$ \\
\hline 5 & $\begin{array}{llll}A & B & C & D\end{array}$ \\
\hline 6 & $C \quad E$ \\
\hline
\end{tabular}

Table 1: A Transaction Database $\mathcal{D}$

\begin{tabular}{c|l|c|c}
\hline Name & Asso. Rules & Support & Confidence \\
\hline$r_{1}$ & $\{A\} \rightarrow\{B\}$ & $3 / 6$ & 1 \\
$r_{2}$ & $\{A\} \rightarrow\{B, C, D\}$ & $3 / 6$ & 1 \\
$r_{3}$ & $\{C\} \rightarrow\{D\}$ & $5 / 6$ & $5 / 6$ \\
$r_{4}$ & $\{C, D\} \rightarrow\{E, F, G\}$ & $2 / 6$ & $2 / 5$ \\
\hline
\end{tabular}

Table 2: Some association rules 
An association rule is a pattern of the form $X \rightarrow Y$ where $X$ (called the antecedent) and $Y$ (called the consequent) are two disjoint itemsets. The interestingness predicate is defined using the notions of support and confidence. The support of an association rule $X \rightarrow Y$ in a transaction database $\mathcal{D}$, defined as $\operatorname{Support}(X \rightarrow Y)=\frac{\operatorname{Support}(X \cup Y)}{|\mathcal{D}|}$, determines how often a rule is applicable to a given dataset, i.e., the occurrence frequency of the rule. The confidence of $X \rightarrow Y$ in $\mathcal{D}$, defined as $\mathcal{C}$ over $(X \rightarrow Y)=\frac{\operatorname{Support}(X \cup Y)}{\text { Support }(X)}$, provides an estimate of the conditional probability of $Y$ given $X$.

A valid association rule is an association rule with support and confidence greater than or equal to the minimum support threshold (minsupp) and minimum confidence threshold (minconf), respectively.

Definition 1 (Mining Association Rules Problem). The problem of mining association rules consists in computing $\operatorname{MAR}(\mathcal{D}$, minsupp, minconf $)=\{X \rightarrow Y \mid X, Y \subseteq \Omega, \operatorname{Support}(X \rightarrow$ $Y) \geqslant \operatorname{minsupp}, \operatorname{Cover}(X \rightarrow Y) \geqslant \operatorname{minconf}\}$

\subsection{SAT-based Non-Redundant Association Rules Mining}

To mine association rules, Boudane et al. [6] proposed a SAT-based approach. Boolean variables are introduced to represent the antecedent and the consequence of an association rule $X \rightarrow$ $Y$. Support and confidence constraints are expressed as pseudo-Boolean constraints over the variables associated to transactions.

Let $\Omega$ be a set of $n$ items, $\mathcal{D}=\left\{\left(1, I_{1}\right), \ldots,\left(m, I_{m}\right)\right\}$ a transaction database, where $\forall i \in$ $\{1, m\}, I_{i} \subseteq \Omega$, minsupp (resp. minconf) a minimum support (resp. confidence) threshold. Each item is associated with two Boolean variables $x_{a}$ and $y_{a} . x_{a}$ (resp. $y_{a}$ ) is true if and only if $a \in X$ (resp. $a \in Y$ ). Similarly to [10], to represent the cover of $X$ and $X \cup Y$, each transaction identifier $i \in\{1, m\}$ is associated with two propositional variables $p_{i}$ and $q_{i} . p_{i}$ (resp. $q_{i}$ ) are used to represent the cover of $X$ (resp. $X \cup Y$ ). More precisely, given a Boolean interpretation $\mathcal{I}$, the corresponding association rule, denoted $r_{\mathcal{I}}$, is $X=\left\{a \in \Omega \mid \mathcal{I}\left(x_{a}\right)=1\right\} \rightarrow$ $Y=\left\{b \in \Omega \mid \mathcal{I}\left(y_{b}\right)=1\right\}$, the cover of $X$ is $\left\{i \in\{1, m\} \mid \mathcal{I}\left(p_{i}\right)=1\right\}$, and the cover of $X \cup Y$ is $\left\{i \in\{1, m\} \mid \mathcal{I}\left(q_{i}\right)=1\right\}$. The SAT encoding of the association rules mining problem is defined by the constraints (27) to (32).

$$
\begin{gathered}
\left(\bigvee_{a \in \Omega} x_{a}\right) \wedge\left(\bigvee_{a \in \Omega} y_{a}\right) \\
\bigwedge_{a \in \Omega}\left(\neg x_{a} \vee \neg y_{a}\right) \\
\bigwedge_{i \in 1 . . m} \neg p_{i} \leftrightarrow \bigvee_{a \in \Omega \backslash I_{i}} x_{a}
\end{gathered}
$$

$$
\begin{gathered}
\bigwedge_{i \in 1 . . m} \neg q_{i} \leftrightarrow \neg p_{i} \vee\left(\bigvee_{a \in \Omega \backslash I_{i}} y_{a}\right) \\
\sum_{i \in 1 . . m} q_{i} \geqslant m \times \operatorname{minsupp} \\
\frac{\sum_{i \in 1 . . m} q_{i}}{\sum_{i \in 1 . . m} p_{i}} \geqslant \text { minconf }
\end{gathered}
$$

The two clauses of the formula (27) express that $X$ and $Y$ are not empty sets. Formula (28) allows to express $X \cap Y=\emptyset$. The formula (29) is used to represent the cover of the itemset $X$ corresponding to the left part of the candidate association rule. We know that the transaction identifier $i$ does not belong to $\operatorname{Cover}(X, \mathcal{D})$ if and only if there exists an item $a \in X$ such that $a \notin I_{i}$. This property is represented by constraint (29) expressing that $p_{i}$ is false if and only if $X$ contains an item that does not belong to the transaction $i$. In the same way, the formula (30) allows to capture the cover of $X \cup Y$. To specify that the support of the candidate rule has to be greater than or equal to the fixed threshold minsupp (in percentage), and the confidence 
is greater than or equal to minconf we use respectively the constraints (31) and (32) expressed by pseudo-Boolean constraints.

To extend the mining task to the closed association rules, the following constraint is added to express that $X \cup Y$ is a closed itemset [10]:

$$
\bigwedge_{a \in \Omega}\left(\left(\bigwedge_{i \in 1 . . m} q_{i} \rightarrow a \in I_{i}\right) \rightarrow x_{a} \vee y_{a}\right)
$$

This formula means that, for all item $a \in \Omega$, if we have $\operatorname{Support}(X \cup Y, \mathcal{D})=\operatorname{Support}(X \cup Y \cup$ $\{a\}, \mathcal{D})$, which is encoded with the formula $\bigwedge_{i \in\{1, m\}} q_{i} \rightarrow a \in I_{i}$, then we get $a \in X \cup Y$, which is encoded with $x_{a} \vee y_{a}$.

Several contributions deal with the enumeration of a compact representation of association rules. Among such representations, one can cite the well-known Minimal Non-Redundant Association Rules [5, 12] defined as follows:

Definition 2 (Minimal Non-Redundant Rule). An association rule $X \rightarrow Y$ is a minimal non-redundant rule iff there is no association rule $X^{\prime} \rightarrow Y^{\prime}$ different from $X \rightarrow Y$ s.t. (1) $\operatorname{Support}(X \rightarrow Y)=\operatorname{Support}\left(X^{\prime} \rightarrow Y^{\prime}\right),(2) \operatorname{Cover}(X \rightarrow Y)=\mathcal{C}$ over $\left(X^{\prime} \rightarrow Y^{\prime}\right)$ and $(3) X^{\prime} \subseteq X$ and $Y \subseteq Y^{\prime}$.

Example 4. Consider the rules given in Table 2. In this set of rules, $\{a\} \rightarrow\{b, c, d\}$ is a minimal non-redundant rule while $\{a\} \rightarrow\{b\}$ is not.

Minimal non-redundant association rules are the closed rules in which the antecedents are minimal w.r.t. set inclusion. The authors of [5] provided a characterization of the antecedents, called minimal generators.

Definition 3 (Minimal Generator). Given a closed itemset $X$. An itemset $X^{\prime} \subseteq X$ is a minimal generator of $X$ iff Support $\left(X^{\prime}\right)=\operatorname{Support}(X)$ and there is no $X^{\prime \prime} \subseteq X$ s.t. $X^{\prime \prime} \subset X^{\prime}$ and $\operatorname{Support}\left(X^{\prime \prime}\right)=\operatorname{Support}(X)$.

In [7], the authors proposed to extend the SAT-based encoding to enumerate the minimal non-redundant association rules. To this end, the SAT-based encoding of association rules mining is enhanced with a Boolean constraint expressing that each antecedent is a minimal generator. This constraint expressing that $\operatorname{Supp}(X \rightarrow Y)=\operatorname{Supp}(X \backslash\{a\} \rightarrow Y)$ is defined as follows:

$$
\left(\bigwedge_{a \in \Omega} x_{a} \rightarrow \bigvee_{\left(i \in\{1 \ldots m\}, a \notin I_{i}\right)}\left(\bigwedge_{b \notin I_{i} \cup\{a\}} \neg x_{b}\right)\right) \vee\left(\sum_{b \in \Omega} x_{b}=1\right)
$$

Using additional variables, this constraint is rewritten as:

$$
\bigwedge_{a \in \Omega}\left(x_{a} \wedge \neg z \rightarrow \bigvee_{\left(a \notin I_{i}\right)} z_{i}\right) \wedge \bigwedge_{i \in 1 . . m}\left(z_{i} \rightarrow \sum_{b \notin I_{i}} x_{b} \leq 1\right) \wedge z \rightarrow\left(\sum_{b \in \Omega} x_{b}=1\right)
$$

As we can observe, the previous constraint (35) involves $m$ conditional AtMostOne constraints. We note $\Phi_{m n r}$, the conjunction of the formulas from (27) to (33) and (35), encoding the problem of minimal non redundant association rules. This encoding is used in our experimental evaluation to show the relevance of our proposed encoding. 


\begin{tabular}{|c|c|c|c|c|}
\hline \multirow[b]{2}{*}{ data (\#items, \#trans, density) } & \multicolumn{2}{|c|}{$\Phi_{n m r}^{n-g a c}$} & \multicolumn{2}{|c|}{$\Phi_{n m r}^{g a c}$} \\
\hline & \#S & $\begin{array}{c}\text { avg. } \\
\text { time(s) }\end{array}$ & $\# \mathrm{~S}$ & $\begin{array}{c}\text { avg } \\
\text { time(s) }\end{array}$ \\
\hline Audiology $(148,216,45 \%)$ & 21 & 855.11 & 22 & $\mathbf{8 5 4 . 8 7}$ \\
\hline Zoo-1 $(36,101,44 \%)$ & 141 & 582.79 & 400 & 0.25 \\
\hline Tic-tac-toe $(27,958,33 \%)$ & 395 & 12.7 & 400 & 0.16 \\
\hline Anneal $(93,812,45 \%)$ & 20 & 855.00 & 252 & 396.65 \\
\hline Australian-credit $(125,653,41 \%)$ & 60 & 765.02 & 288 & 301.96 \\
\hline German-credit $(112,1000,34 \%)$ & 82 & 715.54 & 331 & 203.51 \\
\hline Heart-cleveland $(95,296,47 \%)$ & 100 & 675.02 & 312 & 233.93 \\
\hline Hepatitis $(68,137,50 \%)$ & 102 & 670.51 & 345 & 165.28 \\
\hline Hypothyroid $(88,3247,49 \%)$ & 20 & 855.01 & 128 & 643.01 \\
\hline kr-vs-kp $(73,3196,49 \%)$ & 21 & 852.76 & 173 & 546.40 \\
\hline Lymph $(68,148,40 \%)$ & 21 & 852.75 & 400 & 16.57 \\
\hline Mushroom $(119,8124,18 \%)$ & 20 & 855.08 & 392 & 68.71 \\
\hline Primary-tumor $(31,336,48 \%)$ & 144 & 577.05 & 400 & 3.87 \\
\hline Soybean $(50,650,32 \%)$ & 63 & 758.26 & 400 & 0.72 \\
\hline Vote $(48,435,33 \%)$ & 243 & 353.44 & 400 & 25.34 \\
\hline Splice-1 $(287,3190,21 \%)$ & 363 & 90.68 & 380 & 168.83 \\
\hline Total & 1816 & 645.42 & 5023 & 226.87 \\
\hline
\end{tabular}

Table 3: Comparative results: $\Phi_{n m r}^{n-g a c}$ vs $\Phi_{n m r}^{g a c}$

\section{Experiments}

In this section, we consider the encoding of minimal non-redundant association rules as described by the boolean formula $\Phi_{m n r}$ (see Subsection 4.2). To enumerate the set of models of the resulting CNF formula, we follow the approach of [6]. The proposed model enumeration algorithm is based on a backtrack search DPLL-like procedure. In our experiments, the variables ordering heuristic, focus in priority on the variables of respectively $X$ and $Y$ to select the one to assign next. The main power of this approach consists in using watched literals data structure to perform efficiently the unit propagation process. Let us also note that the constraints (31) and (32) expressing respectively the frequency and the confidence are managed dynamically without translation into CNF form. Indeed, these last constraints are handled and propagated on the fly as usually done in constraint programming. Each model of the propositional formula encoding the association rules mining task corresponds to an association rule obtained by considering the truth values of the propositional variables encoding the antecedent $(X)$ and the consequent $(Y)$ of this rule.

For illustration purposes, in our experiments, $\Phi_{m n r}^{g a c}\left(\right.$ resp. $\left.\Phi_{m n r}^{n_{-} g a c}\right)$ denotes the formula $\Phi_{m n r}$, where the conditional AtMostOne constraints involved in the formula (35) are expressed using the sequential counter encoding (Section 3.1) that maintains (resp. does not maintain) GAC property gac (resp. $n_{-} g a c$ ) expressed by the formula (18) (resp. 17). In our experiments, for each data, the support was varied from $5 \%$ to $100 \%$ with an interval of size $5 \%$. The confidence is varied in the same way. Then, for each data, a set of 400 configurations is generated. All the experiments were done on Intel Xeon quad-core machines with 32GB of RAM running at 2.66 Ghz. For each instance, we fix the timeout to 15 minutes of CPU time.

Table 3 describes our comparative results. We report in column 1 the name of the dataset and its characteristics in parenthesis: number of items (\#items), number of transactions (\#trans) and density. For each encoding, we report the number of solved configurations $(\# S)$, and the average solving time (avg.time in seconds). For each unsolved configuration, the time is set to 900 seconds (time out). In the last row of Table 3, we provide the total number of solved configurations and the global average CPU time in seconds.

As we can observe, the encoding $\Phi_{n m r}^{g a c}$ clearly outperforms $\Phi_{n m r}^{n-g a c}$. Indeed, with the GAC encoding, we solve 3207 more configurations than without maintaining GAC. The encoding $\Phi_{n m r}^{g a c}$ is the best on all the data in terms of the number of solved configurations and average 
CPU time, except for splice-1 data where $\Phi_{n m r}^{n-g a c}$ is better in term of CPU time, but not in the number of solved configurations. Even if we considered a SAT-based data mining application involving only conditional AtMostOne constraints, the performance improvements demonstrate the interest of our proposed encodings.

\section{Conclusion and Future Works}

In this paper, we study the problem of encoding the conditional cardinality constraint of the form $y \rightarrow \sum_{i=1}^{n} x_{i} \leq k$. In this context, we proved that most of the well known GAC encodings of the cardinality constraint need to be adapted in order to maintain such important property. Our contributions suggested that the cardinality constraints should not be considered as an isolated single constraint, but as a part of a more general formula. More generally, encoding a complex constraint into CNF might consider its interaction with the whole formula where such constraint is involved. As an application case, we considered the SAT-based encoding of the minimal non-redundant association rules mining problem, involving several conditional AtMostOne constraints. Experimental results showed that a better encoding of $y \rightarrow \sum_{i=1}^{n} x_{i} \leq$ 1 allows significant performances improvements. As a future work, we plan to extend our framework to tackle other complex conditional constraints, including conditional $0 / 1$ linear inequalities.

\section{References}

[1] R. Asín, R. Nieuwenhuis, A. Oliveras, and E. Rodríguez-Carbonell. Cardinality networks and their applications. In International Conference on Theory and Applications of Satisfiability Testing (SAT), pages 167-180, 2009.

[2] R. Asín, R. Nieuwenhuis, A. Oliveras, and E. Rodríguez-Carbonell. Cardinality networks: a theoretical and empirical study. Constraints, 16(2):195-221, 2011.

[3] O. Bailleux and Y. Boufkhad. Efficient CNF encoding of boolean cardinality constraints. In International Conference on Principles and Practice of Constraint Programming (CP), pages 108122, 2003.

[4] O. Bailleux, Y. Boufkhad, and O. Roussel. New encodings of pseudo-boolean constraints into CNF. In International Conference on Theory and Applications of Satisfiability Testing (SAT), pages 181-194, 2009.

[5] Y. Bastide, N. Pasquier, R. Taouil, G. Stumme, and L. Lakhal. Mining minimal non-redundant association rules using frequent closed itemsets. In Computational Logic, volume 1861, pages 972-986, 2000.

[6] A. Boudane, S. Jabbour, L. Sais, and Y. Salhi. A SAT-based approach for mining association rules. In International Joint Conference on Artificial Intelligence (IJCAI), pages 2472-2478, 2016.

[7] A. Boudane, S. Jabbour, L. Sais, and Y. Salhi. Enumerating non-redundant association rules using satisfiability. In Pacific-Asia Conference on Advances in Knowledge Discovery and Data Mining (PAKDD), pages 824-836, 2017.

[8] N. Eén and N. Sörensson. Translating pseudo-boolean constraints into SAT. Journal on Satisfiability, Boolean Modeling and Computation, 2(1-4):1-26, 2006.

[9] A. Erez and A. Nadel. Finding bounded path in graph using SMT for automatic clock routing. In Computer Aided Verification - 27th International Conference, CAV 2015, San Francisco, CA, USA, July 18-24, 2015, Proceedings, Part II, pages 20-36, 2015. 
[10] S. Jabbour, L. Sais, and Y. Salhi. The Top-k frequent closed itemset mining using Top-k SAT problem. In European Conference on Machine Learning and Principles and Practice of Knowledge Discovery in Databases (ECML PKDD), pages 403-418, 2013.

[11] S. Jabbour, L. Saïs, and Y. Salhi. A pigeon-hole based encoding of cardinality constraints. In International Symposium on Artificial Intelligence and Mathematics (ISAIM), 2014.

[12] M. Kryszkiewicz. Representative association rules and minimum condition maximum consequence association rules. In European Conference on Principles of Data Mining and Knowledge Discovery (PKDD), pages 361-369, 1998.

[13] J. P. Marques-Silva and I. Lynce. Towards robust cnf encodings of cardinality constraints. In International Conference on Principles and Practice of Constraint Programming (CP), pages 483497, 2007.

[14] D. A. Plaisted and S. Greenbaum. A structure-preserving clause form translation. J. Symb. Comput., 2(3):293-304, Sept. 1986.

[15] C. Sinz. Towards an optimal CNF encoding of boolean cardinality constraints. In International Conference on Principles and Practice of Constraint Programming (CP), pages 827-831, 2005.

[16] G. Tseitin. On the complexity of derivations in the propositional calculus. In H. Slesenko, editor, Structures in Constructives Mathematics and Mathematical Logic, Part II, pages 115-125, 1968.

[17] J. P. Warners. A linear-time transformation of linear inequalities into conjunctive normal form. Information Processing Letters, 1996. 